\title{
Construction of non-subadditive measures and discretization of Borel measures
}

\author{
by
}

\author{
Johan F. A a r nes (Trondheim)
}

\begin{abstract}
The main result of the paper provides a method for construction of regular non-subadditive measures in compact Hausdorff spaces. This result is followed by several examples. In the last section it is shown that "discretization" of ordinary measures is possible in the following sense. Given a positive regular Borel measure $\lambda$, one may construct a sequence of non-subadditive measures $\mu_{n}$, each of which only takes a finite set of values, and such that $\mu_{n}$ converges to $\lambda$ in the $w^{*}$-topology.
\end{abstract}

1. Introduction. In this paper we continue the study of non-subadditive measures undertaken in [1], [2] and [5], called there "quasi-measures". They are set-functions defined on the open and on the closed subsets of a locally compact Hausdorff space $X$, and represent a genuine generalization of regular Borel measures in such spaces. This paper is devoted to showing how they arise and may be constructed when $X$ is compact, and to giving some applications.

Non-subadditive measures (NSA-measures), as the name indicates, are generally not subadditive. Indeed, if they are, then they turn out to be ordinary regular Borel measures. This lack of subadditivity is what makes NSA-measures different, and in some respects more interesting than ordinary measures. Instead of weighing effects or events on an additive scale, the NSA-measures register a cumulative effect of events. To produce a certain result, several other results must occur simultaneously. This is of course a very superficial description, and only future development and applications can substantiate what we indicate here.

Even if NSA-measures by definition are generalizations of ordinary measures, their existence is not an obvious matter, and turns out to be closely linked to properties of the underlying topological space. The existence of NSA-measures was first established in the author's paper [1]. In [2] we gave

1991 Mathematics Subject Classification: 28C15. 
a description of the basic properties of extremal NSA-measures (those taking only the values 0 and 1). In [5] Knudsen gave a procedure for the construction of such extremal NSA-measures in certain spaces. The present paper is devoted to the construction of general NSA-measures. Our main result (Theorem 5.1) shows how all NSA-measures arise and may be constructed (in certain spaces). We believe that even when applied to ordinary measures this is a new result of some interest. The main result is followed by several examples of construction. In the last section of the paper we show that "discretization" of ordinary measures is possible in the following sense: Given a positive, regular Borel measure $\lambda$ (for instance Lebesgue measure on the unit sphere in $\mathbb{R}^{3}$ ), we construct a sequence of NSA-measures $\mu_{n}$, each of which takes only a finite set of values, and such that $\mu_{n}$ converges to $\lambda$ in the $w^{*}$-topology.

1.1. Notation and basic concepts. Throughout $X$ denotes a compact Hausdorff space and $\mathbf{A}=C(X)$ is the algebra of real-valued continuous functions on $X$. For $a \in \mathbf{A}$ we let $\mathbf{A}(a)$ denote the smallest uniformly closed subalgebra of $\mathbf{A}$ containing $a$ and 1. A function $\varrho: \mathbf{A} \rightarrow \mathbb{R}$ satisfying $\varrho(1)=1, \varrho(a) \geq 0$ if $a \geq 0$ and such that $\varrho$ is linear on $\mathbf{A}(a)$ for each $a \in \mathbf{A}$ is called a non-linear state (previously called a quasi-state).

Let $\mathcal{C}$ denote the collection of closed subsets of $X$, let $\mathcal{O}$ denote the collection of open subsets of $X$ and put $\mathcal{A}=\mathcal{C} \cup \mathcal{O}$. A real-valued, nonnegative function $\mu$ on $\mathcal{A}$ is called a NSA-measure in $X$ if the following conditions are satisfied:

(Q0) $\mu(K)+\mu(X \backslash K)=\mu(X)$ for $K \in \mathcal{C}$,

(Q1) $K_{1} \subset K_{2} \Rightarrow \mu\left(K_{1}\right) \leq \mu\left(K_{2}\right)$ for $K_{1}, K_{2} \in \mathcal{C}$,

(Q2) $K_{1} \cap K_{2}=\emptyset \Rightarrow \mu\left(K_{1} \cup K_{2}\right)=\mu\left(K_{1}\right)+\mu\left(K_{2}\right)$ for $K_{1}, K_{2} \in \mathcal{C}$,

(Q3) $\mu(U)=\sup \{\mu(K): K \subset U, K \in \mathcal{C}\}$ for $U \in \mathcal{O}$.

$\mu$ is normalized if $\mu(X)=1$. For simplicity we shall assume that all NSA-measures in this paper are normalized.

R e m ark. A NSA-measure $\mu$ which is also subadditive is called a regular content. In Halmos $([4], \S 54$, Theorem A) it is shown that a regular content has a unique extension to a regular Borel measure in $X$.

In [1] we established that there is a 1-1 correspondence between nonlinear states and normalized NSA-measures. The set of all non-linear states is a convex set, denoted by $Q$, which is compact in the topology of pointwise convergence on $\mathbf{A}$.

A subset $D$ of $X$ is co-connected if $X \backslash D$ is connected. $D$ is solid if it is connected and co-connected.

In what follows a subscript s indicates "solid", and a subscript c indicates "connected", so that for instance $\mathcal{A}_{\mathrm{s}}$ is the collection of all solid sets that 
are either open or closed and $\mathcal{C}_{\mathrm{c}}$ is the family of closed connected sets. $\mathcal{C}_{0}$ is the family of closed sets with only a finite number of connected components. $\mathcal{O}_{0}=\left\{U \in \mathcal{O}: X \backslash U \in \mathcal{C}_{0}\right\}$, and $\mathcal{A}_{0}=\mathcal{C}_{0} \cup \mathcal{O}_{0}$.

2. Fundamentals. In this section we introduce the main object of study in this paper: the solid set-function. Some preliminaries are needed. From now on we assume that $X$ is a compact Hausdorff space which is connected and locally connected. By convention the empty set $\emptyset$ is connected, so $\emptyset$ and $X$ both belong to $\mathcal{C}_{\mathrm{s}}$.

Definition 2.1. A partition of $X$ is a collection of mutually disjoint, non-void sets $\left\{A_{i}\right\}_{i \in I} \subseteq \mathcal{A}_{\mathrm{s}}$, where at most finitely many of the $A_{i}$ are closed, and such that $X=\bigcup_{i \in I} A_{i}$. The number of closed sets in a partition $\mathcal{P}$ is called the order of $\mathcal{P}$.

Any connected and locally connected space $X$ with more than one point has a partition. For if $x \in X$, then $\{x\}^{\mathrm{c}}$ is open and has a non-void connected component $V \in \mathcal{O}_{\mathrm{s}}$ (cf. Lemma 3.2 in the next section). Hence $C=V^{\mathrm{c}} \in \mathcal{C}_{\mathrm{s}}$ and $\{C, V\}$ is a partition of $X$ of order 1. Accordingly, partitions of order 1 are called trivial.

Let $\left\{A_{i}\right\}_{i \in I}$ be a non-trivial partition, and let $I_{0}=\left\{i \in I: A_{i}\right.$ is closed $\}$.

Definition 2.2. $\left\{A_{i}\right\}_{i \in I}$ is irreducible if the following two conditions hold:

(i) $\bigcup_{i \in I_{0}} A_{i}$ is not co-connected.

(ii) For any proper subset $I^{\prime}$ of $I_{0}, \bigcup_{i \in I^{\prime}} A_{i}$ is co-connected.

Necessarily, any irreducible partition has order $\geq 2$, and any partition of order 2 is irreducible. For a given space $X$, let $n$ denote the maximal order of any irreducible partition. If $n$ is finite, let $g=n-1$. If $X$ only permits trivial partitions, put $g=0$.

Definition 2.3. A function $\mu: \mathcal{A}_{\mathrm{s}} \rightarrow[0,1]$ satisfying the conditions (A), (B) and (C) below is called a solid set-function.

(A) For any finite collection of disjoint sets $\left\{C_{1}, \ldots, C_{n}\right\} \subseteq \mathcal{C}_{\mathrm{s}}$ such that $C_{j} \subseteq C \in \mathcal{C}_{\mathrm{s}}$ for $j=1, \ldots, n$ we have

$$
\sum_{j=1}^{n} \mu\left(C_{j}\right) \leq \mu(C) .
$$

(B) For all $U \in \mathcal{O}_{\text {s }}$ we have

$$
\mu(U)=\sup \left\{\mu(C): C \subseteq U, C \in \mathcal{C}_{\mathrm{s}}\right\} .
$$


(C) For any trivial or irreducible partition $\left\{A_{i}\right\}_{i \in I}$ of $X$ we have

$$
\sum_{i \in I} \mu\left(A_{i}\right)=1 .
$$

Note. If $g=0$ then $X$ has no irreducible partitions, so (C) reduces to the condition that $\mu(A)+\mu\left(A^{\mathrm{c}}\right)=1$ for each set $A \in \mathcal{A}_{\mathrm{s}}$.

Remark. It is important to realize that the restriction of a NSAmeasure $\mu$ to $\mathcal{A}_{\mathrm{s}}$ is always a solid set-function. Indeed, since $\mu$ is additive and monotone on $\mathcal{C},(\mathrm{A})$ is clearly true. If $K$ is closed and contained in a solid open set $U$, there is a solid closed set $C$ such that $K \subseteq C \subseteq U$ (see Section 3), and (B) follows. Finally, (C) is a consequence of Proposition 2.1 of [1] and Corollary 2.1 of [2].

We next point out that any NSA-measure (or any regular Borel measure) is uniquely determined by its restriction to $\mathcal{C}_{\mathrm{s}}$. For suppose $\mu$ is a NSAmeasure in $X$. If the values of $\mu$ on $\mathcal{C}_{\mathrm{s}}$ are known, then they are also known on the complements of these sets, i.e. on $\mathcal{O}_{\mathrm{s}}$. But then, by virtue of Corollary 2.1 quoted above, and Lemma 3.2 of this paper, it follows that $\mu$ is determined on the family of closed connected sets, and hence also on $\mathcal{C}_{0}$. Now let $K$ be a closed set contained in an open set $U$, and let $U=\bigcup_{i \in I} U_{i}$ be the decomposition of $U$ into its connected components. By compactness of $K$ there is a finite index set $I^{\prime} \subseteq I$ such that $K \subseteq \bigcup_{i \in I^{\prime}} U_{i}$. Let $K_{i}=K \cap U_{i}$ $\left(i \in I^{\prime}\right)$. By Lemma 3.1 of this paper there are connected closed sets $C_{i}$ such that $K_{i} \subseteq C_{i} \subseteq U_{i}$ for each $i \in I^{\prime}$. But then $C=\bigcup C_{i}$ belongs to $\mathcal{C}_{0}$ and $K \subseteq C \subseteq U$. By (Q3) in the definition of NSA-measures it therefore follows that $\mu$ is determined on the open sets by the values it takes on the class $\mathcal{C}_{0}$. Taking complements again we see that the uniqueness claim follows.

ExAmple. Let $X=S^{2}$ and let $p_{1}, \ldots, p_{5}$ be five distinct points in $X$. For $C \in \mathcal{C}_{\mathrm{s}}$ define $\mu(C)$ to be 0 if $C$ contains at most one of these points, to be $1 / 2$ if $C$ contains two or three of the points, and to be 1 if $C$ contains four or five points. It is easily seen that (A) and (B) hold, and (C) is true because $S^{2}$ only permits trivial partitions.

The main purpose of this paper is to show that a solid set-function has a unique extension to a NSA-measure in $X$. In the example just given this extension turns out to be an extreme point in the convex set of all normalized NSA-measures in $X$ (cf. [2], Example 2.1).

To begin with, we record some of the basic properties of solid setfunctions.

Proposition 2.1. If $\mu$ is a solid set-function in $X$ then

1. $\mu(\emptyset)=0, \mu(X)=1$.

2. $A_{1} \subseteq A_{2} \Rightarrow \mu\left(A_{1}\right) \leq \mu\left(A_{2}\right)$ for all $A_{1}, A_{2} \in \mathcal{A}_{\mathrm{s}}$. 
3. If $U_{1}, \ldots, U_{n}$ belong to $\mathcal{O}_{\mathrm{s}}$, where $n$ is an arbitrary integer $\geq 1$, $U_{i} \cap U_{j}=\emptyset, i \neq j$, and $U_{i} \subseteq U \in \mathcal{O}_{\text {s }}$ for all $i=1, \ldots, n$, then

$$
\sum_{i=1}^{n} \mu\left(U_{i}\right) \leq \mu(U)
$$

The proof is left to the reader.

Rem ark. Let $\mu$ be a solid set-function in $X$, and suppose $\left\{C_{i}\right\}_{i \in I} \subseteq \mathcal{C}_{\mathrm{s}}$ is an arbitrary collection of mutually disjoint sets. From (A) it immediately follows (let $C=X$ ) that the subfamily $\left\{C_{i}: \mu\left(C_{i}\right)>0\right\}$ is at most countably infinite. When it comes to summation we may therefore replace the index set $I$ by $\mathbb{Z}^{+}$or $\mathbb{N}$. This is implicit when we write $\sum_{i \in I} \mu\left(C_{i}\right)$. If $C_{i} \subseteq C \in \mathcal{C}_{\mathrm{s}}$ for all $i \in I$ we must clearly have $\sum_{i \in I} \mu\left(C_{i}\right) \leq \mu(C)$. Analogously, in view of Proposition 2.1.3 the corresponding statements hold for open sets, and will be utilised without further comments.

For later use we include the following:

LEMma 2.1. Let $\mathcal{F}=\left\{C_{j}\right\}_{j \in J} \subseteq \mathcal{C}_{\mathrm{s}}(n \geq 1)$ be a finite family of mutually disjoint sets such that $\bigcup_{j \in J} C_{j}$ is not co-connected. Then $\mathcal{F}$ has a subfamily $\mathcal{F}^{\prime}$ such that each connected component $U_{i}$ of $U=\left(\bigcup\left\{C_{j} \in \mathcal{F}^{\prime}\right\}\right)^{\mathrm{c}}$ belongs to $\mathcal{O}_{\mathrm{s}}(i \in I=$ some index set $)$ and $\mathcal{F}^{\prime} \cup\left\{U_{i}\right\}_{i \in I}$ is an irreducible partition of $X$.

Proof. $\mathcal{F}$ contains a subfamily $\mathcal{F}^{\prime}=\left\{C_{j}\right\}_{j \in J^{\prime}}$ such that $U=\left(\bigcup_{j \in J^{\prime}} C_{j}\right)^{\mathrm{c}}$ is not connected, but for any proper subset $J^{\prime \prime}$ of $J^{\prime}$, the set $\left(\bigcup_{j \in J^{\prime \prime}} C_{j}\right)^{\mathrm{c}}$ is connected. Let $U=\bigcup_{i \in I} U_{i}$ be the decomposition of $U$ into its connected components. Then card $I \geq 2$ and each $U_{i}$ is in $\mathcal{O}_{c}$. We claim that each $U_{i}$ is also co-connected. So pick an arbitrary $U_{0} \in\left\{U_{i}\right\}_{i \in I}$, and let $I^{\prime}=I \backslash\{0\}$. We must show that $U_{0}^{\mathrm{c}}=\left(\bigcup_{j \in J^{\prime}} C_{j}\right) \cup\left(\bigcup_{i \in I^{\prime}} U_{i}\right)$ is connected. Suppose not. Then there are non-void, disjoint, closed sets $K_{1}$ and $K_{2}$ in $X$ such that $K_{1} \cup K_{2}=U_{0}^{\mathrm{c}}$. By connectedness, $K_{1}$ (and $K_{2}$ ) must contain all or nothing of each of the sets $C_{j}\left(j \in J^{\prime}\right)$ and $U_{i}\left(i \in I^{\prime}\right)$. Let

$$
J_{k}=\left\{j \in J^{\prime}: C_{j} \subseteq K_{k}\right\}, \quad I_{k}=\left\{i \in I^{\prime}: U_{i} \subseteq K_{k}\right\}, \quad k=1,2 .
$$

We must have $J_{1} \neq \emptyset$ and $J_{2} \neq \emptyset$ and at least one of the sets $I_{k}$ is nonempty since card $I \geq 2$. Suppose $I_{1} \neq \emptyset$. Since $K_{1}$ is closed, its complement $K_{1}^{\mathrm{c}}=K_{2} \cup U_{0}$ is open. But then also

$$
V=\left(K_{2} \cup U_{0}\right) \cup\left(\bigcup_{i \in I_{1}} U_{i}\right)
$$

is open and not connected. But $V^{\mathrm{c}}=\bigcup_{j \in J_{1}} C_{j}$, which is a contradiction, since $J_{1}$ is a proper subset of $J^{\prime}$. Hence $U_{0}^{\mathrm{c}}$ is connected and the proof is complete. 
Proposition 2.2. For a space $X$ we have $g=0$ if and only if $\bigcup\{C \in \mathcal{F}\}$ is co-connected for any finite family $\mathcal{F} \subseteq \mathcal{C}_{\mathrm{s}}$ of disjoint sets.

Proof. This follows from Lemma 2.1.

Remark. In [5] Knudsen has shown that if $X$ is also locally pathwise connected and $H^{1}(X, \mathbb{Q})=(0)$ then $g=0$. Hence $S^{n}$ (the unit $n$-sphere) and $B^{n}$ (the unit $n$-ball) have $g=0$, the torus and an annulus in the plane have $g=1$, etc.

3. Topological preliminaries. The results in this section are more or less known. Some proofs are included for the convenience of the reader. Good references are [3] and [6].

Throughout we assume that $X$ is a compact Hausdorff space which is connected and locally connected.

Lemma 3.1. Let $K \in \mathcal{C}, U \in \mathcal{O}$ and $K \subseteq U$. If either $K$ or $U$ is connected, then there is a set $V \in \mathcal{O}_{\text {c }}$ such that $K \subseteq V \subseteq \bar{V} \subseteq U$.

Lemma 3.2. Let $K \in \mathcal{C}_{\mathrm{c}}$. Then each connected component of $V=X \backslash K$ belongs to $\mathcal{O}_{\mathrm{s}}$.

Lemma 3.3. Let $K \in \mathcal{C}_{\mathrm{s}}, U \in \mathcal{O}$ and $K \subseteq U$. Then there is a set $V \in \mathcal{O}_{\mathrm{s}}$ such that $K \subseteq V \subseteq \bar{V} \subseteq U$.

Proof of Lemma 3.3. By Lemma 3.1 we may assume that $U$ is connected. Let $F=X \backslash U$, so $F \subseteq X \backslash K$, which is open and connected.

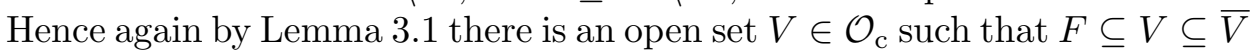
and $\bar{V} \cap K=\emptyset$. Let $W=X \backslash \bar{V}$ so $K \subseteq W \subseteq U$. Since $\bar{V} \in \mathcal{C}_{\mathrm{c}}$ it now follows that each connected component of $W$ belongs to $\mathcal{O}_{\text {s }}$ (Lemma 3.2), and $K$ must be contained in exactly one of them. The proof is complete.

3.1. The solid hull of a closed connected set. Let $K \in \mathcal{C}_{\mathrm{c}}, V \in \mathcal{O}_{\mathrm{s}}$ and suppose $K \subseteq V$. Let $X \backslash K=W=\bigcup_{i \in I} W_{i}$ be the decomposition of $W$ into disjoint connected components. Then $C=X \backslash V$ is connected and is contained in one of them, say $W_{0}$, called the exterior component of $W$ with respect to $V$. Each $W_{i}$ with $i \neq 0$ is then called an interior component. Since each $W_{i}$ is in $\mathcal{O}_{\text {s }}$ the set $\widetilde{K}=X \backslash W_{0}$ belongs to $\mathcal{C}_{\mathrm{s}}$. We have

$$
\widetilde{K}=\bigcup\left\{W_{i}: i \neq 0\right\} \cup K
$$

and $K \subseteq \widetilde{K} \subseteq V . \widetilde{K}$ is called the solid hull of $K$ with respect to $V$.

Lemma 3.4. Suppose $C_{1}, C_{2}$ are disjoint, closed, connected sets, both contained in $V \in \mathcal{O}_{\mathrm{s}}$. Then either 
(i) $\widetilde{C}_{1} \cap \widetilde{C}_{2}=\emptyset$, or

(ii) $\widetilde{C}_{1} \subseteq \widetilde{C}_{2}$ or $\widetilde{C}_{2} \subseteq \widetilde{C}_{1}$ (proper inclusion).

Proof. Let $W^{j}=X \backslash C_{j}(j=1,2)$, and let $W^{j}=\bigcup_{i \in I_{j}} W_{i}^{j}$ as above, with $W_{0}^{j}$ as the exterior component of $W^{j}$ with respect to $V$. Now $C_{1} \cap C_{2}=\emptyset$ so

$$
C_{1} \subseteq W^{2} \quad \text { and } \quad C_{2} \subseteq W^{1} .
$$

Since $C_{1}$ and $C_{2}$ are connected, there are unique components $W_{i}^{2}$ and $W_{k}^{1}$ such that $C_{1} \subseteq W_{i}^{2}$ and $C_{2} \subseteq W_{k}^{1}$.

(i) Suppose $C_{1} \subseteq W_{0}^{2}$ and $C_{2} \subseteq W_{0}^{1}$. Then $C_{2} \subseteq \widetilde{C}_{2}=X \backslash W_{0}^{2} \subseteq W^{1}$ by the first inclusion. Since $\widetilde{C}_{2}$ is connected the second inclusion implies that $\widetilde{C}_{2} \subseteq W_{0}^{1}=X \backslash \widetilde{C}_{1}$, so $\widetilde{C}_{1} \cap \widetilde{C}_{2}=\emptyset$.

(ii) Suppose $C_{2} \subseteq W_{k}^{1}$ for some $k \neq 0$. Then $W_{k}^{1} \subseteq V$ so $X \backslash V \subseteq$ $X \backslash W_{k}^{1} \subseteq W^{2}$. Since $X \backslash W_{k}^{1}$ is connected and contains $X \backslash V$ we must have $X \backslash W_{k}^{1} \subseteq W_{0}^{2}$, so that $\widetilde{C}_{2}=X \backslash W_{0}^{2} \subseteq W_{k}^{1} \subseteq \widetilde{C}_{1}$. Similarly, if $C_{1} \subseteq W_{i}^{2}$ for some $i \neq 0$, then $\widetilde{C}_{1} \subseteq \widetilde{C}_{2}$. The proof is complete.

Remark. We may note that in the above situation one of the sets is always contained in the exterior component associated with the other set.

4. Extension to $\mathcal{C}_{0}$. Let $\mu$ be a solid set-function. Our goal in this section is to extend $\mu$ to a function on $\mathcal{C}_{0}$ to $[0,1]$ such that

$(\mathrm{Q} 1)_{0} K_{1} \subseteq K_{2} \Rightarrow \mu\left(K_{1}\right) \leq \mu\left(K_{1}\right)$ for $K_{1}, K_{2} \in \mathcal{C}_{0}$.

$\left.(\mathrm{Q} 2)_{0} K_{1} \cap K_{2}=\emptyset \Rightarrow \mu\left(K_{1} \cup K_{2}\right)=\mu\left(K_{1}\right)+\mu K_{2}\right)$ for $K_{1}, K_{2} \in \mathcal{C}_{0}$.

(Q3) $)_{0}$ For all $C \in \mathcal{C}_{0}$ and all $\varepsilon>0$ there is $C^{\prime} \in \mathcal{C}_{0}$ such that $C \cap C^{\prime}=\emptyset$ and $\mu(C)+\mu\left(C^{\prime}\right)>1-\varepsilon$.

If $K \in \mathcal{C}_{\mathrm{c}}$ we know by Lemma 3.2 that $X \backslash K=V=\bigcup_{i \in I} V_{i}$, where the sets $V_{i}$ are open, connected, co-connected and mutually disjoint. From Proposition 2.1.3 it therefore follows that the set $\left\{V_{i}: \mu\left(V_{i}\right)>0\right\}$ is at most countable, and that $\sum_{i \in I} \mu\left(V_{i}\right) \leq 1$. We may then define

$$
\mu(K)=1-\sum_{i \in I} \mu\left(V_{i}\right) .
$$

Next, if $K=K_{1} \cup \ldots \cup K_{n} \in \mathcal{C}_{0}$, where the $K_{j}$ are connected and mutually disjoint, we put $\mu(K)=\sum_{j=1}^{n} \mu\left(K_{j}\right)$.

Proposition 4.1. The function $\mu$ as defined above maps $\mathcal{C}_{0}$ into $[0,1]$ and satisfies conditions $(\mathrm{Q} 1)_{0},(\mathrm{Q} 2)_{0}$ and $(\mathrm{Q} 3)_{0}$.

We start with (Q1) $)_{0}$ If $K_{1}, K_{2} \in \mathcal{C}_{0}$, then $K_{1} \subseteq K_{2} \Rightarrow \mu\left(K_{1}\right) \leq \mu\left(K_{2}\right)$.

Suppose first that $K_{1}$ is connected, while $K_{2}=C_{1} \cup \ldots \cup C_{n}$ with the $C_{j}$ in $\mathcal{C}_{\mathrm{c}}$ and mutually disjoint. Then we must have $K_{1} \subseteq C_{j}$ for some $j$, say 
$K_{1} \subseteq C_{1}$. Let $\bigcup_{i \in I} U_{i}=X \backslash K_{1}$ and $\bigcup_{i \in J} V_{j}=X \backslash C_{1}$ be the decompositions of these sets into their solid components. Each $V_{j}$ must be contained in some $U_{i}$. Let

$$
J_{i}=\left\{j \in J: V_{j} \subseteq U_{i}\right\} .
$$

Then $\bigcup_{i \in I} J_{i}=J$, and by Proposition 2.1.3 we get

$$
\sum_{j \in J} \mu\left(V_{j}\right)=\sum_{i \in I} \sum_{j \in J_{i}} \mu\left(V_{j}\right) \leq \sum_{i \in I} \mu\left(U_{i}\right) .
$$

Hence $\mu\left(K_{1}\right)=1-\sum_{i \in I} \mu\left(U_{i}\right) \leq 1-\sum_{j \in J} \mu\left(V_{j}\right) \leq \mu\left(C_{1}\right)$.

Next, assume that $K_{1}=C_{1}^{\prime} \cup \ldots \cup C_{m}^{\prime}$ is the decomposition of $K_{1}$ into its connected components. Since $K_{1} \subseteq K_{2}$ each $C_{i}^{\prime}$ is contained in $C_{j}$ for some $j \in\{1, \ldots, n\}$.

Suppose $C_{i_{1}}^{\prime}, \ldots, C_{i_{k}}^{\prime} \subseteq C_{j}$. We need to show that $\sum_{l=1}^{k} \mu\left(C_{i_{l}}^{\prime}\right) \leq \mu\left(C_{j}\right)$. To simplify the notation, assume that $C_{0}, C_{1}, \ldots, C_{n}$ are connected, closed, disjoint and contained in $C \in \mathcal{C}_{\mathrm{c}}$. Let $X \backslash C_{0}=V=\bigcup_{i \in I} V_{i}$. Since all the $C_{j}$ are connected, each $C_{j}$ for $j \geq 1$ is contained in some $V_{i}$, and only finitely many $V_{i}$ will contain some $C_{j}$. Let

$$
J_{i}=\left\{j: C_{j} \subseteq V_{i}\right\}, \quad i \in I .
$$

Let $U=X \backslash C=\bigcup_{i \in K} U_{k}$. Then $U \subseteq V$ and we put

$$
K_{i}=\left\{k \in K: U_{k} \subseteq V_{i}\right\} .
$$

The $U_{k}$ are disjoint and obviously also disjoint from all the $C_{j}$. We want to show that

$$
\sum_{j=0}^{n} \mu\left(C_{j}\right) \leq \mu(C)
$$

which is equivalent to

$$
1-\sum_{i \in I} \mu\left(V_{i}\right)+\sum_{j=1}^{n} \mu\left(C_{j}\right) \leq 1-\sum_{k \in K} \mu\left(U_{k}\right),
$$

or

$$
\sum_{j=1}^{n} \mu\left(C_{j}\right)+\sum_{k \in K} \mu\left(U_{k}\right) \leq \sum_{i \in I} \mu\left(V_{i}\right) .
$$

Now (4.3) will follow if we can show that

$$
\sum_{j \in J_{i}} \mu\left(C_{j}\right)+\sum_{k \in K_{i}} \mu\left(U_{k}\right) \leq \mu\left(V_{i}\right)
$$

for all $i \in I$. Therefore, what we really need is the following result, formally stated as a lemma. 
Lemma A. Let $C_{1}, \ldots, C_{n} \in \mathcal{C}_{\mathrm{c}}$ and $U_{1}, \ldots, U_{k}, \ldots \in \mathcal{O}_{\mathrm{s}}$ be mutually disjoint subsets of $V \in \mathcal{O}_{\mathrm{s}}$. Then

$$
\sum_{j=1}^{n} \mu\left(C_{j}\right)+\sum_{k=1}^{\infty} \mu\left(U_{k}\right) \leq \mu(V) .
$$

Proof. We employ induction on $n$.

Step 1: $n=1$. Let $X \backslash C_{1}=W=\bigcup_{i \in I} W_{i}$. Now $X \backslash V$ is connected and contained in $W$, and is therefore contained in one of $W$ 's components, which we denote by $W_{0}$, i.e. $X \backslash V \subseteq W_{0}$ and $W_{0}$ is the exterior component of $W$ with respect to $V$ (cf. Section 3). Let $I^{\prime}=I \backslash\{0\}$ and put $\widetilde{C}_{1}=$ $C_{1} \cup\left(\bigcup_{i \in I^{\prime}} W_{i}\right)=X \backslash W_{0}$, i.e. $\widetilde{C}_{1}$ is the solid hull of $C_{1}$ with respect to $V$. We want to show that

$$
\mu\left(C_{1}\right)+\sum_{k=1}^{\infty} \mu\left(U_{k}\right) \leq \mu(V)
$$

or

$$
1-\sum_{i \in I^{\prime}} \mu\left(W_{i}\right)-\mu\left(W_{0}\right)+\sum_{k=1}^{\infty} \mu\left(U_{k}\right) \leq \mu(V),
$$

i.e.

$$
\mu\left(\widetilde{C}_{1}\right)+\sum_{k=1}^{\infty} \mu\left(U_{k}\right) \leq \mu(V)+\sum_{i \in I^{\prime}} \mu\left(W_{i}\right)
$$

We have $U_{k} \subseteq W$ for all $k=1,2, \ldots$ (since all $U_{k}$ are disjoint from $C_{1}$ ) and we let

$$
K_{i}=\left\{k \in \mathbb{Z}^{+}: U_{k} \subseteq W_{i}\right\}, \quad i \in I .
$$

Then we have, using Proposition 2.1.3 again,

$$
\begin{aligned}
\sum_{k=1}^{\infty} \mu\left(U_{k}\right) & =\sum_{k \in K_{0}} \mu\left(U_{k}\right)+\sum_{i \in I^{\prime}}\left[\sum_{k \in K_{i}} \mu\left(U_{k}\right)\right] \\
& \leq \sum_{k \in K_{0}} \mu\left(U_{k}\right)+\sum_{i \in I^{\prime}} \mu\left(W_{i}\right) .
\end{aligned}
$$

Hence (4.6) will follow if we can show that

$$
\mu\left(\widetilde{C}_{1}\right)+\sum_{k \in K_{0}} \mu\left(U_{k}\right) \leq \mu(V),
$$

where we observe that if $k \in K_{0}$ then $U_{k}$ is disjoint from $\widetilde{C}_{1}$. In turn, (4.7) will follow from the next lemma (which in fact is just a weakened version of Lemma A). 
Lemma B. Let $C_{1}, \ldots, C_{m} \in \mathcal{C}_{\mathrm{s}}$ and $U_{1}, \ldots, U_{k}, \ldots \in \mathcal{O}_{\mathrm{s}}$ be mutually disjoint subsets of $V \in \mathcal{O}_{\mathrm{s}}$. Then

$$
\sum_{j=1}^{m} \mu\left(C_{j}\right)+\sum_{k=1}^{\infty} \mu\left(U_{k}\right) \leq \mu(V) .
$$

Proof. Let $\varepsilon>0$ be arbitrary. Select $N \in \mathbb{Z}^{+}$such that $\sum_{k>N} \mu\left(U_{k}\right)<$ $\varepsilon / 2$. For $k=1, \ldots, N$ take $C_{k}^{\prime} \subseteq U_{k}$ such that $C_{k}^{\prime} \in \mathcal{C}_{\mathrm{s}}$ and $\mu\left(C_{k}^{\prime}\right)>$ $\mu\left(U_{k}\right)-\varepsilon / 2^{k+1}$ by $(\mathrm{B})$. Then

$$
\begin{aligned}
\sum_{k=1}^{\infty} \mu\left(U_{k}\right) & =\sum_{k=1}^{N} \mu\left(U_{k}\right)+\sum_{k>N} \mu\left(U_{k}\right) \\
& <\sum_{k=1}^{N} \mu\left(C_{k}^{\prime}\right)+\varepsilon \sum_{k=1}^{N} \frac{1}{2^{k+1}}+\frac{\varepsilon}{2}<\sum_{k=1}^{N} \mu\left(C_{k}^{\prime}\right)+\varepsilon .
\end{aligned}
$$

Let $C=X \backslash V$, so $C \in \mathcal{C}_{\mathrm{s}}$ and $C \cap C_{j}=C \cap C_{k}^{\prime}=\emptyset$ for all $j$ and $k$. Then by $(\mathrm{A})$ we get

$$
\sum_{j=1}^{m} \mu\left(C_{j}\right)+\sum_{k=1}^{N} \mu\left(C_{k}^{\prime}\right)+\mu(C) \leq 1
$$

Hence

$$
\sum_{j=1}^{m} \mu\left(C_{j}\right)+\sum_{k=1}^{\infty} \mu\left(U_{k}\right) \leq \mu(V)+\varepsilon .
$$

Since $\varepsilon>0$ was arbitrary, the assertion follows.

The inequality (4.7) now follows (taking $m=1$ ) since $\widetilde{C}_{1} \in \mathcal{C}_{\mathrm{s}}$. We have now established Lemma A for $n=1$.

Step 2. Suppose inductively that Lemma A has been verified for $k=$ $1, \ldots, n-1$. For $j=1, \ldots, n$ let $X \backslash C_{j}=W^{j}=\bigcup\left\{W_{i}^{j}: i \in I_{j}\right\}$, and let $W_{0}^{j}$ be the exterior component of $W^{j}$ with respect to $V$. Let $\widetilde{C}_{j}=X \backslash W_{0}^{j}$ denote the solid hull of $C_{j}$ with respect to $V$.

Case 1: $\widetilde{C}_{j} \cap \widetilde{C}_{l}=\emptyset$ for all $j, l$ with $j \neq l$. The assertion (4.5) is equivalent to

$$
\sum_{j=1}^{n} \mu\left(\widetilde{C}_{j}\right)+\sum_{k=1}^{\infty} \mu\left(U_{k}\right) \leq \mu(V)+\sum_{j=1}^{n} \sum_{i \in I_{j}^{\prime}} \mu\left(W_{i}^{j}\right),
$$

where $I_{j}^{\prime}=I_{j} \backslash\{0\}$. Now observe that $X \backslash \bigcup_{j=1}^{n} \widetilde{C}_{j}=\bigcap_{j=1}^{n} W_{0}^{j}$, so that if 
we put

$$
\begin{aligned}
& K_{0}=\left\{k \in \mathbb{Z}^{+}: U_{k} \subseteq \bigcap_{j=1}^{n} W_{0}^{j}\right\}, \\
& K_{j}=\left\{k \in \mathbb{Z}^{+}: U_{k} \subseteq \widetilde{C}_{j}\right\}, \quad j=1, \ldots, n,
\end{aligned}
$$

then we exhaust all possibilities for the sets $U_{k}$ in mutually exclusive cases.

By Lemma B we now get

$$
\sum_{j=1}^{n} \mu\left(\widetilde{C}_{j}\right)+\sum_{k \in K_{0}} \mu\left(U_{k}\right) \leq \mu(V) .
$$

If $U_{k} \subseteq \widetilde{C}_{j}$ then $U_{k} \subseteq W_{i}^{j}$ for some $i \in I_{j}^{\prime}$ so that (using Proposition 2.1.3 again)

$$
\sum_{k \in K_{j}} \mu\left(U_{k}\right) \leq \sum_{i \in I_{j}^{\prime}}\left(W_{i}^{j}\right), \quad j=1, \ldots, n .
$$

Combining (4.10) and (4.11) we obtain (4.9), which establishes the assertion (4.5) in Case 1.

Case 2: $\widetilde{C}_{j} \cap \widetilde{C}_{j} \neq \emptyset$ for some pair $j \neq l$. By Lemma 3.4 either $\widetilde{C}_{j} \subseteq \widetilde{C}_{l}$ or $\widetilde{C}_{l} \subseteq \widetilde{C}_{j}$ (proper inclusion). We may therefore re-index the sets $C_{j}(j=$ $1, \ldots, n)$ as follows:

1. $\widetilde{C}_{10}, \widetilde{C}_{20}, \ldots, \widetilde{C}_{m 0}(m<n)$ are mutually disjoint.

2. $\widetilde{C}_{j l} \subseteq \widetilde{C}_{j 0}$ for $l=0, \ldots, m_{j}$ and $1 \leq j \leq m$, where the inclusions are proper if $l \geq 1$.

3. $m+\sum_{j=1}^{m} m_{j}=n$.

The verification of this is a simple induction argument based on Lemma 3.4, and is left to the reader. We return to the proof of (4.9), which after the re-indexing takes the form

$$
\sum_{j=1}^{m} \sum_{l=0}^{m_{j}} \mu\left(\widetilde{C}_{j l}\right)+\sum_{k=1}^{\infty} \mu\left(U_{k}\right) \leq \mu(V)+\sum_{j=1}^{m} \sum_{l=0}^{m_{j}} \sum_{i \in I_{j l}^{\prime}} \mu\left(W_{i}^{j l}\right),
$$

where $X \backslash C_{j l}=\bigcup\left\{W_{i}^{j l}: i \in I_{j l}\right\}$.

Let $K_{0}, K_{1}, \ldots, K_{n}$ be defined as in Case 1 . Then again by Lemma B we immediately get

$$
\sum_{j=1}^{m} \mu\left(\widetilde{C}_{j 0}\right)+\sum_{k \in K_{0}} \mu\left(U_{k}\right) \leq \mu(V)
$$


so to establish (4.12) it remains to show that

$$
\sum_{j=1}^{m} \sum_{l=1}^{m_{j}} \mu\left(\widetilde{C}_{j l}\right)+\sum_{j=1}^{m} \sum_{k \in K_{j}} \mu\left(U_{k}\right) \leq \sum_{j=1}^{m} \sum_{l=0}^{m j} \sum_{i \in I_{j l}^{\prime}} \mu\left(W_{i}^{j l}\right),
$$

which will follow if we can show that for each $j=1, \ldots, m$ we have

$$
\sum_{l=1}^{m_{j}} \mu\left(\widetilde{C}_{j l}\right)+\sum_{k \in K_{j}} \mu\left(U_{k}\right) \leq \sum_{l=0}^{m j} \sum_{i \in I_{j l}^{\prime}} \mu\left(W_{i}^{j l}\right) .
$$

Now $\mu\left(\widetilde{C}_{j l}\right)=\mu\left(C_{j l}\right)+\sum_{i \in I_{j l}^{\prime}} \mu\left(W_{i}^{j l}\right)$, so (4.15) is equivalent to

$$
\sum_{l=1}^{m j} \mu\left(C_{j l}\right)+\sum_{k \in K_{j}} \mu\left(U_{k}\right) \leq \sum_{i \in I_{j 0}^{\prime}} \mu\left(W_{i}^{j 0}\right) .
$$

Let

$$
\begin{aligned}
& J_{j i}=\left\{l \leq m_{j}: C_{j l} \subseteq W_{i}^{j 0}\right\}, \quad i \in I_{j 0}^{\prime}, \\
& K_{j i}=\left\{k \in K_{j}: U_{k} \subseteq W_{i}^{j 0}\right\}, \quad i \in I_{j 0}^{\prime} .
\end{aligned}
$$

Since $C_{j l} \subseteq W_{i}^{j 0}$ for some $i \in I_{j 0}^{\prime}$ if $1 \leq l \leq m_{j}$, and $U_{k} \subseteq W_{i}^{j 0}$ for some $i \in I_{j 0}^{\prime}$ if $k \in K_{j}$, we exhaust all possibilities for $l$ and $k$ in this manner. Hence (4.16) will follow if we can show that for each $i \in I_{j 0}^{\prime}$,

$$
\sum_{l \in J_{j i}} \mu\left(C_{j l}\right)+\sum_{k \in K_{j i}} \mu\left(U_{k}\right) \leq \mu\left(W_{i}^{j 0}\right) .
$$

The number of elements in $J_{j i}$ is $\leq m_{j} \leq m<n$ so (4.17) now follows by the induction hypothesis. This concludes the proof of Lemma A.

This also concludes the proof of condition (Q1) $)_{0}$ in Proposition 4.1. As for (Q2) $)_{0}$, if $K_{1}, K_{2} \in \mathcal{C}_{0}$ and $K_{1} \cap K_{2}=\emptyset$ let $K_{1}=C_{1} \cup \ldots \cup C_{n}$ and $K_{2}=C_{1}^{\prime} \cup \ldots \cup C_{m}^{\prime}$ be their decompositions into connected components. Then $K_{1} \cup K_{2}=C_{1} \cup \ldots \cup C_{n} \cup C_{1}^{\prime} \cup \ldots \cup C_{m}^{\prime}$ and the right-hand side represents the decomposition of $K_{1} \cup K_{2}$ into connected components. From the definition of $\mu$ on $\mathcal{C}_{0}$ it now immediately follows that $\mu\left(K_{1} \cup K_{2}\right)=\mu\left(K_{1}\right)+\mu\left(K_{2}\right)$, and $(\mathrm{Q} 2)_{0}$ follows.

It remains to verify the regularity property $(\mathrm{Q} 3)_{0}$.

Let $K \in \mathcal{C}_{0}$ be arbitrary. Then $K=C_{0} \cup C_{1} \cup \ldots \cup C_{n}$, where $C_{j} \in \mathcal{C}_{\mathrm{c}}$ and $C_{j} \cap C_{k}=\emptyset$ if $j \neq k$. Let $X \backslash C_{0}=V=\bigcup_{i \in I} V_{i}$, where the $V_{i}$ are mutually disjoint and belong to $\mathcal{O}_{\mathrm{s}}$. Each $C_{j}(j=1, \ldots, n)$ is contained in some $V_{i}$, and we let

$$
J_{i}=\left\{j \in\{1, \ldots, n\}: C_{j} \subseteq V_{i}\right\}, \quad i \in I .
$$

From Lemma A it follows that $\sum_{j \in J_{i}} \mu\left(C_{j}\right) \leq \mu\left(V_{i}\right)$ for all $i \in I$. Let $\varepsilon>0$ be arbitrary, and choose $N$ such that $\sum_{i>N} \mu\left(V_{i}\right)<\varepsilon / 2$ (since 
$\left\{i \in I: \mu\left(V_{i}\right)>0\right\}$ is countable, we may assume that $\left.I=\mathbb{Z}^{+}\right)$. Now suppose for the moment that there are sets $C_{i}^{\prime} \in \mathcal{C}_{0}$ for $i=1, \ldots, N$ such that

$$
\left\{\begin{array}{l}
C_{i}^{\prime} \subseteq V_{i} \text { and } C_{i}^{\prime} \cap C_{j}=\emptyset \text { if } j \in J_{i} \\
\mu\left(C_{i}^{\prime}\right)>\mu\left(V_{i}\right)-\sum_{j \in J_{i}} \mu\left(C_{j}\right)-\varepsilon / 2^{i+1} .
\end{array}\right.
$$

Then let $C^{\prime}=\bigcup_{i=1}^{N} C_{i}^{\prime}$, so $C^{\prime} \in \mathcal{C}_{0}$ and $C^{\prime} \cap K=\emptyset$. Moreover (by (Q2) 0 ),

$$
\begin{aligned}
\mu\left(C^{\prime}\right) & =\sum_{i=1}^{N} \mu\left(C_{i}^{\prime}\right)>\sum_{i=1}^{N} \mu\left(V_{i}\right)-\sum_{i=1}^{N} \sum_{j \in J_{i}} \mu\left(C_{j}\right)-\sum_{i=1}^{N} \frac{\varepsilon}{2^{i+1}} \\
& >\sum_{i=1}^{\infty} \mu\left(V_{i}\right)-\frac{\varepsilon}{2}-\sum_{j=1}^{n} \mu\left(C_{j}\right)-\frac{\varepsilon}{2} \\
& =1-\mu\left(C_{0}\right)-\sum_{j=1}^{n} \mu\left(C_{j}\right)-\varepsilon=1-\mu(K)-\varepsilon,
\end{aligned}
$$

which establishes $(\mathrm{Q} 3)_{0}$ under the assumption that (4.18) holds. So what we need is the following.

Lemma C. Let $C_{1}, \ldots, C_{n} \in \mathcal{C}_{\mathrm{c}}$ be mutually disjoint and contained in $V \in \mathcal{O}_{\mathrm{s}}$. For each $\varepsilon>0$ there is a $C^{\prime} \in \mathcal{C}_{0}$ such that $C^{\prime} \cap C_{j}=\emptyset$ for all $j$, $C^{\prime} \subseteq V$ and

$$
\sum_{j=1}^{n} \mu\left(C_{j}\right)+\mu\left(C^{\prime}\right)>\mu(V)-\varepsilon .
$$

Proof. We first consider the special case where all the sets $C_{1}, \ldots, C_{n}$ belong to $\mathcal{C}_{\mathrm{s}}$, and $V=X$. The proof in this case goes by induction on $n$. For $n=1$ the assertion is covered by (B) in the definition of a solid set-function. Now assume $n \geq 2$ and that the assertion is true for all $k<n$. Let $\varepsilon>0$ be arbitrary. Combining (B) and Lemma 3.3, we may find a family of mutually disjoint sets $V_{1}, \ldots, V_{n}$ in $\mathcal{O}_{\mathrm{s}}$ such that $C_{j} \subseteq V_{j}$ and

$$
\mu\left(V_{j}\right)<\mu\left(C_{j}\right)+\varepsilon / n, \quad j=1, \ldots, n .
$$

Next, choose $W_{j} \in \mathcal{O}_{\mathrm{s}}$ such that

$$
C_{j} \subseteq W_{j} \subseteq \bar{W}_{j} \subseteq V_{j}, \quad j=1, \ldots, n
$$

(Lemma 3.3). Let $F_{j}$ be the solid hull of $\bar{W}_{j}$ with respect to $V_{j}$. Then

$$
C_{j} \subseteq W_{j} \subseteq F_{j} \subseteq V_{j}, \quad F_{j} \in \mathcal{C}_{\mathrm{s}},
$$

for $j=1, \ldots, n$.

At this point we distinguish between two subcases:

(i) $U=X \backslash \bigcup_{j=1}^{n} F_{j}$ is connected. Then $C^{\prime}=\bar{U}$ is connected and contained in $X \backslash \bigcup_{j=1}^{n} W_{j}$, so $C^{\prime} \cap C_{j}=\emptyset$ for $j=1, \ldots, n$. On the other hand, 
$X \backslash C^{\prime} \subseteq \bigcup_{j=1}^{n} V_{j}$. If $X \backslash C^{\prime}=\bigcup_{i \in I} O_{i}$ is the decomposition of $X \backslash C^{\prime}$ into its disjoint connected components, then each $O_{i}$ belongs to $\mathcal{O}_{\mathrm{s}}$ and each $O_{i}$ is contained in some $V_{j}$. But then, by Proposition 2.3.3, it follows that $\sum_{i \in I} \mu\left(O_{i}\right) \leq \sum_{j=1}^{n} \mu\left(V_{j}\right)$. Consequently, we get

$$
\mu\left(C^{\prime}\right)=1-\sum_{i \in I} \mu\left(O_{i}\right) \geq 1-\sum_{j=1}^{n} \mu\left(V_{j}\right)>1-\sum_{j=1}^{n}\left(C_{j}\right)-\varepsilon,
$$

which yields the assertion in this case.

(ii) $X \backslash \bigcup_{j=1}^{n} F_{j}$ is not connected. Let $m$ be the minimal number of sets in any subcollection $\mathcal{F}^{\prime} \subseteq \mathcal{F}=\left\{F_{1}, \ldots, F_{n}\right\}$ such that $\bigcup\left\{F_{j}: F_{j} \in \mathcal{F}^{\prime}\right\}$ is not co-connected. Let $\mathcal{F}_{1}$ be such a subfamily of $\mathcal{F}$ with $m$ elements, $2 \leq m \leq n$. Let $U=\left(\bigcup\left\{F_{j}: F_{j} \in \mathcal{F}_{1}\right\}\right)^{\mathrm{c}}$. By Lemma 2.1 we have $U=\bigcup_{i \in I} U_{i}, U_{i}$ disjoint, $U_{i} \in \mathcal{O}_{\mathrm{s}}$. Again we distinguish between two cases:

a) $m=n$. Let $N$ be such that $\sum_{i>N} \mu\left(U_{i}\right)<\varepsilon / 2$. Then for $i \leq N$ choose $K_{i} \subseteq U_{i}$ such that $K_{i} \in \mathcal{C}_{\mathrm{s}}$ and

$$
\mu\left(K_{i}\right)>\mu\left(U_{i}\right)-\frac{\varepsilon}{2 N} .
$$

The family $\left\{F_{j}, U_{i}: j=1, \ldots, n, i \in I\right\}$ is an irreducible partition of $X$ so that by $(\mathrm{C})$,

$$
\sum_{j=1}^{n} \mu\left(F_{j}\right)+\sum_{i \in I} \mu\left(U_{i}\right)=1
$$

and hence

$$
\begin{aligned}
\sum_{j=1}^{n} \mu\left(C_{j}\right)+\sum_{i=1}^{N} \mu\left(K_{i}\right) & >\sum_{j=1}^{n} \mu\left(F_{j}\right)-\varepsilon+\sum_{i=1}^{N} \mu\left(U_{i}\right)-\varepsilon \\
& >\sum_{j=1}^{n} F_{j}+\sum_{i \in I} \mu\left(U_{i}\right)-\frac{5 \varepsilon}{2}=1-\frac{5 \varepsilon}{2},
\end{aligned}
$$

which proves the assertion when we take $C^{\prime}=\bigcup_{i=1}^{N} K_{i}$.

b) $m<n$. The family $\left\{F_{j}, U_{i}: j=1, \ldots, m, i \in I\right\}$ is an irreducible partition of $X$. If $F_{j} \notin \mathcal{F}_{1}$ then $F_{j} \subseteq U_{i}$ for some $i \in I$. Since $m \geq 2$ we have $n-m<n-1$, so that for any $i \in I$ the collection

$$
\mathcal{F}_{i}=\left\{F_{k} \in \mathcal{F}: F_{k} \subseteq U_{i}\right\} \cup\left\{X \backslash U_{i}\right\}
$$

will have at most $n-1$ elements. By the induction hypothesis it therefore follows that there is a $K_{i} \in \mathcal{C}_{0}$ such that $F_{k} \cap K_{i}=\emptyset$ for all $F_{k} \in \mathcal{F}_{i}, K_{i} \subseteq U_{i}$ and

$$
\sum_{F_{k} \in \mathcal{F}_{i}} \mu\left(F_{k}\right)+\mu\left(K_{i}\right)+\mu\left(X \backslash U_{i}\right)>1-\frac{\varepsilon}{N},
$$


SO

$$
\sum_{F_{k} \in \mathcal{F}_{i}} \mu\left(F_{k}\right)+\mu\left(K_{i}\right)>\mu\left(U_{i}\right)-\frac{\varepsilon}{N}, \quad i=1, \ldots, N
$$

Then we get

$$
\begin{aligned}
\sum_{j=1}^{n} \mu\left(C_{j}\right)+\sum_{i=1}^{N} \mu\left(K_{i}\right) & >\sum_{j=1}^{n} \mu\left(F_{j}\right)-\varepsilon+\sum_{i=1}^{N} \mu\left(K_{i}\right) \\
& \geq \sum_{j=1}^{m} \mu\left(F_{j}\right)+\sum_{i=1}^{N} \sum_{F_{k} \in \mathcal{F}_{i}} \mu\left(F_{k}\right)+\sum_{i=1}^{N} \mu\left(K_{i}\right)-\varepsilon \\
& >\sum_{j=1}^{m} \mu\left(F_{j}\right)+\sum_{i=1}^{N} \mu\left(U_{i}\right)-2 \varepsilon \\
& >\sum_{j=1}^{m} \mu\left(F_{j}\right)+\sum_{i \in I} \mu\left(U_{i}\right)-\frac{5 \varepsilon}{2}=1-\frac{5 \varepsilon}{2},
\end{aligned}
$$

which proves the assertion in case b).

Finally, if $C_{1}, \ldots, C_{n} \subseteq V \in \mathcal{O}_{\text {s }}$, let $C_{0}=X \backslash V$. Applying the argument above to the family $C_{0}, C_{1}, \ldots, C_{n}$ now yields Lemma $\mathrm{C}$ when all the sets $C_{1}, \ldots, C_{n}$ are solid.

Next, we turn to the general case, assuming that all the sets $C_{j}$ $(j=1, \ldots, n)$ belong to $\mathcal{C}_{\mathrm{c}}$. Again we use induction on $n$.

Step 1: $n=1$. Let $C \subseteq V \in \mathcal{O}_{\mathrm{s}}$ and $C \in \mathcal{C}_{\mathrm{c}}$. Let $X \backslash C=\bigcup_{i \in I} W_{i}$ with $W_{i} \in \mathcal{O}_{\mathrm{s}}$. Let $W_{0}$ be the exterior component with respect to $V$, and put $\widetilde{C}=X \backslash W_{0}$ as usual. We have $\widetilde{C} \subseteq V$ and by the first part of the proof, there is a $K_{0} \in \mathcal{C}_{0}$ such that $\widetilde{C} \cap K_{0}=\emptyset, K_{0} \subseteq V$ and $\mu(\widetilde{C})+$ $\mu\left(K_{0}\right)>\mu(V)-\varepsilon / 2$. Choose $N$ such that $\sum_{i>N} \mu\left(W_{i}\right)<\varepsilon / 4$ (restricting to a countable subfamily again). Next, choose $K_{i} \subseteq W_{i}$ such that $K_{i} \in \mathcal{C}_{\mathrm{s}}$ and $\mu\left(K_{i}\right)>\mu\left(W_{i}\right)-\varepsilon /\left(4 \cdot 2^{i}\right)$ for $i=1, \ldots, N$. All the $K_{i}(i=0,1, \ldots, N)$ are now disjoint from $C$, mutually disjoint $\left(K_{0} \subseteq W_{0} \cap V\right)$ and contained in $V$. Take $K=\bigcup_{i=0}^{N} K_{i}$, so $K \in \mathcal{C}_{0}, K \cap C=\emptyset$ and $K \subseteq V$. Moreover,

$$
\begin{aligned}
\mu(K) & =\sum_{i=0}^{N} \mu\left(K_{i}\right)>\mu(V)-\mu(\widetilde{C})-\frac{\varepsilon}{2}+\sum_{i=1}^{N} \mu\left(W_{i}\right)-\frac{\varepsilon}{4} \\
& >\mu(V)-\left(1-\mu\left(W_{0}\right)\right)+\sum_{i \in I} \mu\left(W_{i}\right)-\varepsilon \\
& =\mu(V)-\mu(C)-\varepsilon,
\end{aligned}
$$

which establishes the assertion for $n=1$.

S t e p 2: Assume inductively that the assertion is true for $k=1, \ldots, n-1$. 
Case 1: $\widetilde{C}_{1}, \ldots, \widetilde{C}_{n}$ are mutually disjoint. By the first part of the proof there is $K_{0} \in \mathcal{C}_{0}$ such that $K_{0} \subseteq V, K_{0} \cap \widetilde{C}_{j}=\emptyset(j=1, \ldots, n)$ and $\mu\left(K_{0}\right)>$ $\mu(V)-\sum_{j=1}^{n} \mu\left(\widetilde{C}_{j}\right)-\varepsilon / 2$.

Let $X \backslash C_{j}=W^{j}=\bigcup_{i \in I_{j}} W_{i}^{j}$, where $W_{0}^{j}$ is the exterior component of $W^{j}$ with respect to $V$ (i.e. $\widetilde{C}_{j}=X \backslash W_{0}^{j}$ ). Let $N_{j} \in \mathbb{Z}^{+}$be such that $\sum_{i>N_{j}} \mu\left(W_{i}^{j}\right)<\varepsilon /(4 n)$. For $i=1, \ldots, N_{j}$ pick $K_{i}^{j} \subseteq W_{i}^{j}$ such that $K_{i}^{j} \in \mathcal{C}_{\mathrm{s}}$ and

$$
\mu\left(K_{i}^{j}\right)>\mu\left(W_{i}^{j}\right)-\frac{\varepsilon}{4 n} \cdot \frac{1}{2^{i}}, \quad j=1, \ldots, n .
$$

Since the $\widetilde{C}_{j}$ are mutually disjoint, all the sets $K_{i}^{j}$ are mutually disjoint and also disjoint from $K_{0} \subseteq \bigcap_{j=1}^{n} W_{0}^{j}$. Let $K=K_{0} \cup \bigcup\left\{K_{i}^{j}: 1 \leq i \leq N_{j}, j=\right.$ $1, \ldots, n\}$ so $K \in \mathcal{C}_{0}, K \cap C_{j}=\emptyset(j=1, \ldots, n)$ and $K \subseteq V$. We now obtain

$$
\begin{aligned}
\mu(K) & =\mu\left(K_{0}\right)+\sum_{j=1}^{n} \sum_{i=1}^{N_{j}} \mu\left(K_{i}^{j}\right) \\
& >\mu(V)-\sum_{j=1}^{n} \mu\left(\widetilde{C}_{j}\right)-\frac{\varepsilon}{2}+\sum_{j=1}^{n} \sum_{i=1}^{N_{j}}\left[\mu\left(W_{i}^{j}\right)-\frac{\varepsilon}{4 n} \cdot \frac{1}{2^{i}}\right] \\
& >\mu(V)-\sum_{j=1}^{n}\left[\mu\left(\widetilde{C}_{j}\right)-\sum_{i \in I_{j}^{\prime}} \mu\left(W_{i}^{j}\right)\right]-\varepsilon \\
& =\mu(V)-\sum_{j=1}^{n} \mu\left(C_{j}\right)-\varepsilon,
\end{aligned}
$$

which proves the assertion in Case 1 .

Case 2: $\widetilde{C}_{j} \cap \widetilde{C}_{k} \neq \emptyset$ for some pair $j \neq k$. We proceed as in the proof of Lemma $\mathrm{A}$, and re-index the sets $C_{1}, \ldots, C_{n}$ so that

1. $\widetilde{C}_{10}, \widetilde{C}_{20}, \ldots, \widetilde{C}_{m 0}(m<n)$ are mutually disjoint.

2. $\widetilde{C}_{j l} \subseteq \widetilde{C}_{j 0}$ for $l=0, \ldots, m_{j}$ and $1 \leq j \leq m$, where the inclusions are proper if $l \geq 1$.

3. $m+\sum_{j=1}^{m} m_{j}=n$.

As before, let $X \backslash C_{j l}=\bigcup\left\{W_{i}^{j l}: i \in I_{j l}\right\}=W^{j l}$, and let $W_{0}^{j l}$ denote the exterior component of $W^{j l}$ with respect to $V$. Again, by the first part of the proof, there is $K_{0} \in \mathcal{C}_{0}$ such that $K_{0} \subseteq V, K_{0} \cap \widetilde{C}_{j 0}=\emptyset(j=1, \ldots, m)$, and

$$
\mu\left(K_{0}\right)>\mu(V)-\sum_{j=1}^{m} \mu\left(\widetilde{C}_{j 0}\right)-\frac{n-m}{n} \varepsilon .
$$

For each $j \in\{1, \ldots, m\}$ choose $N_{j} \in \mathbb{Z}^{+}$such that $\sum_{i>N_{j}} \mu\left(W_{i}^{j 0}\right)<\varepsilon /(2 n)$. 
When $l \geq 1$ each set $C_{j l}$ is contained in some interior component $W_{i}^{j 0}$ of $W^{j 0}$, i.e. $i \neq 0$. We let

$$
J_{j i}=\left\{l: 1 \leq l \leq m_{j}, C_{j l} \subseteq W_{i}^{j 0}\right\}, \quad 1 \leq i \leq N_{j} .
$$

If $J_{j i}=\emptyset$ there is by (B) a set $K_{i}^{j} \subseteq W_{i}^{j 0}$ such that $K_{i}^{j} \in \mathcal{C}_{\mathrm{s}}$ and

$$
\mu\left(K_{i}^{j}\right)>\mu\left(W_{i}^{j 0}\right)-\frac{\varepsilon}{2 N_{j} n} .
$$

If $J_{j i} \neq \emptyset$ there is, by the induction hypothesis, since $m_{j}<n$, a set $K_{i}^{j} \subseteq$ $W_{i}^{j 0}$ such that $K_{i}^{j} \in \mathcal{C}_{0}$ and

$$
\mu\left(K_{i}^{j}\right)>\mu\left(W_{i}^{j 0}\right)-\sum_{l \in J_{j i}} \mu\left(C_{j l}\right)-\frac{\varepsilon}{2 N_{j} n} .
$$

Let $K_{j}=\bigcup_{i=1}^{N_{j}} K_{i}^{j} \in \mathcal{C}_{0}$ for $j=1, \ldots, m$. Then, adding the inequalities (4.20) and (4.21), we get

$$
\begin{aligned}
\mu\left(K_{j}\right) & =\sum_{i=1}^{N_{j}} \mu\left(K_{i}^{j}\right) \\
& >\sum_{i=1}^{N_{j}} \mu\left(W_{i}^{j 0}\right)-\sum_{i=1}^{N_{j}} \sum_{l \in J_{j i}} \mu\left(C_{j l}\right)-\frac{\varepsilon}{2 n} \\
& >\sum_{i=1}^{\infty} \mu\left(W_{i}^{j 0}\right)-\sum_{l=1}^{m_{j}} \mu\left(C_{j l}\right)-\frac{\varepsilon}{n} \\
& =\mu\left(\widetilde{C}_{j 0}\right)-\mu\left(C_{j 0}\right)-\sum_{l=1}^{m_{j}} \mu\left(C_{j l}\right)-\frac{\varepsilon}{n},
\end{aligned}
$$

so

$$
\mu\left(K_{j}\right)>\mu\left(\widetilde{C}_{j 0}\right)-\sum_{l=0}^{m_{j}} \mu\left(C_{j l}\right)-\frac{\varepsilon}{n}, \quad j=1, \ldots, n .
$$

Now let $K=\bigcup_{j=0}^{m} K_{j} \in \mathcal{C}_{0}$. By addition of (4.19) and the equations (4.22) we get

$$
\begin{aligned}
\mu(K)= & \sum_{j=0}^{m} \mu\left(K_{j}\right) \\
> & \mu(V)-\sum_{j=1}^{m} \mu\left(C_{j 0}\right)-(n-m) \frac{\varepsilon}{n} \\
& +\sum_{j=1}^{m} \mu\left(\widetilde{C}_{j 0}\right)-\sum_{j=1}^{m} \sum_{l=0}^{m_{j}} \mu\left(C_{j l}\right)-\frac{m \varepsilon}{n} \\
= & \mu(V)-\sum_{j=1}^{m} \sum_{l=0}^{m_{j}} \mu\left(C_{j l}\right)-\varepsilon,
\end{aligned}
$$


which concludes the proof of the lemma since the double sum above comprises all the sets $C_{1}, \ldots, C_{n}$ given initially.

Lemma C was what was needed to establish (4.18) and the proof of Proposition 4.1 is therefore also complete.

5. Extension to a NSA-measure. Let $X$ be a compact Hausdorff space which is connected and locally connected. Suppose $\mu: \mathcal{C}_{0} \rightarrow[0,1]$ satisfies $(\mathrm{Q} 1)_{0},(\mathrm{Q} 2)_{0}$ and $(\mathrm{Q} 3)_{0}$ of the preceding section. If $V \in \mathcal{O}_{0}$ we may define

$$
\mu(V)=1-\mu(X \backslash V),
$$

thereby obtaining a function $\mu: \mathcal{A}_{0} \rightarrow[0,1]$ which satisfies the conditions (Q0), (Q1), (Q2) and (Q3) for a NSA-measure, as given in the introduction. In particular, we may note that (Q3) is an immediate consequence of (Q3) 0 and (5.1), i.e. we have, for $V \in \mathcal{O}_{0}$,

$$
\mu(V)=\sup \left\{\mu(K): K \subseteq V, K \in \mathcal{C}_{0}\right\} .
$$

We now use (5.2) to extend $\mu$ to all of $\mathcal{O}$, and then define

$$
\mu(C)=1-\mu(X \backslash C)
$$

for an arbitrary set $C \in \mathcal{C}$.

Proposition 5.1. The function $\mu: \mathcal{A} \rightarrow[0,1]$, as defined by (5.2) for $V \in \mathcal{O}$ and by (5.3) for $C \in \mathcal{C}$, is a NSA-measure in $X$.

Proof. Our task is to verify that (Q0)-(Q3) hold on $\mathcal{A}$. (Q0) is automatic by (5.3). From (5.2) it follows that $\mu\left(V_{1}\right) \leq \mu\left(V_{2}\right)$ if $V_{1} \subseteq V_{2}$ and $V_{1}, V_{2} \in \mathcal{O}$. (Q1) now follows from (5.3). To prove (Q3) we first observe that if $K \subseteq U, K \in \mathcal{C}, U \in \mathcal{O}$, then there is a set $C \in \mathcal{C}_{0}$ such that $K \subseteq C \subseteq U$. This follows as in the remark subsequent to the definition of a solid set-function. (Q3) now follows from (5.2) and (Q1).

To prove (Q2) we first show that $\mu$ is additive on $\mathcal{O}$. Let $U_{1}$ and $U_{2}$ be disjoint open sets, and let $\varepsilon>0$ be arbitrary. Choose $K \in \mathcal{C}_{0}$ such that $K \subseteq U_{1} \cup U_{2}$ and $\mu(K)>\mu\left(U_{1} \cup U_{2}\right)-\varepsilon$.

Next, choose $K_{1}, K_{2} \in \mathcal{C}_{0}$ such that $K_{i} \subseteq U_{i}, \mu\left(K_{i}\right)>\mu\left(U_{i}\right)-\varepsilon(i=1,2)$. Then we get, since $K_{1} \cap K_{2}=\emptyset$,

$$
\begin{aligned}
\mu\left(U_{1}\right)+\mu\left(U_{2}\right) & <\mu\left(K_{1}\right)+\mu\left(K_{2}\right)+2 \varepsilon=\mu\left(K_{1} \cup K_{2}\right)+2 \varepsilon \\
& \leq \mu\left(U_{1} \cup U_{2}\right)+2 \varepsilon<\mu(K)+3 \varepsilon \\
& =\mu\left(K_{1}^{\prime} \cup K_{2}^{\prime}\right)+3 \varepsilon=\mu\left(K_{1}^{\prime}\right)+\mu\left(K_{2}^{\prime}\right)+3 \varepsilon \\
& \leq \mu\left(U_{1}\right)+\mu\left(U_{2}\right)+3 \varepsilon,
\end{aligned}
$$

where $K_{i}^{\prime}=K \cap U_{i} \in \mathcal{C}_{0}(i=1,2)$. Since $\varepsilon>0$ was arbitrary it follows that 
if $U_{1} \cap U_{2}=\emptyset$ and $U_{1}, U_{2} \in \mathcal{O}$, then

$$
\mu\left(U_{1} \cup U_{2}\right)=\mu\left(U_{1}\right)+\mu\left(U_{2}\right) .
$$

We are now ready to show that $\mu$ is additive on $\mathcal{C}$. Let $K_{1} \cap K_{2}=\emptyset$ and $K_{1}, K_{2} \in \mathcal{C}$. Combining (5.2) and (5.3) we find, for $\varepsilon>0$ arbitrary, sets $U, U_{1}, U_{2}$ in $\mathcal{O}$ such that

$$
\begin{aligned}
& K_{1} \cup K_{2} \subseteq U, \quad \mu(U)<\mu\left(K_{1} \cup K_{2}\right)+\varepsilon, \\
& K_{i} \subseteq U_{i}, \quad \mu\left(U_{i}\right)<\mu\left(K_{i}\right)+\varepsilon, \quad i=1,2 .
\end{aligned}
$$

Since $\mu$ is monotone on $\mathcal{O}$ we may assume that $U_{1} \cap U_{2}=\emptyset$. Then we get

$$
\begin{aligned}
\mu\left(K_{1}\right)+\mu\left(K_{2}\right) & >\mu\left(U_{1}\right)+\mu\left(U_{2}\right)-2 \varepsilon=\mu\left(U_{1} \cup U_{2}\right)-2 \varepsilon \\
& \geq \mu\left(K_{1} \cup K_{2}\right)-2 \varepsilon \quad(\text { by }(\mathrm{Q} 3)) \\
& >\mu(U)-3 \varepsilon \\
& \geq \mu\left(\left(U \cap U_{1}\right) \cup\left(U \cap U_{2}\right)\right)-3 \varepsilon \\
& =\mu\left(U \cap U_{1}\right)+\mu\left(U \cap U_{1}\right)-3 \varepsilon \\
& \geq \mu\left(K_{1}\right)+\mu\left(K_{2}\right)-3 \varepsilon .
\end{aligned}
$$

Since $\varepsilon>0$ was arbitrary it follows that if $K_{1}, K_{2} \in \mathcal{C}$ and $K_{1} \cap K_{2}=\emptyset$, then

$$
\mu\left(K_{1}\right)+\mu\left(K_{2}\right)=\mu\left(K_{1} \cup K_{2}\right),
$$

which is (Q2).

Remark. In the author's paper [1] another version of the extension from $\mathcal{C}_{0}$ to $\mathcal{C}$ is given, which avoids the connectedness assumptions on $X$ ([1], Proposition 5.1). However, a different assumption has to be made, and the present treatment is closer in spirit to the rest of this paper.

Combining the last result with the results of Section 4 we have our main result:

TheOREM 5.1. Let $X$ be a compact Hausdorff space which is connected and locally connected.

(i) Any solid set-function $\mu: \mathcal{A}_{\mathrm{s}} \rightarrow[0,1]$ has a unique extension to a NSA-measure in $X$.

(ii) The arising NSA-measure is extremal if and only if $\mu: \mathcal{A}_{\mathrm{s}} \rightarrow\{0,1\}$.

(iii) If $\mu$ is subadditive on $\mathcal{C}_{\mathrm{c}}$, i.e. if $\mu\left(C_{1} \cup C_{2}\right) \leq \mu\left(C_{1}\right)+\mu\left(C_{2}\right)$ for all $C_{1}, C_{2} \in \mathcal{C}_{\mathrm{c}}$, then $\mu$ extends to a uniquely determined regular Borel measure in $X$.

Part (ii) is obvious and the proof of the third part is left to the reader. One has to show that $\mu$ is subadditive on $\mathcal{C}$ and then apply a standard result of measure theory (cf. the remark in the introduction). 
As a first application of the theorem above we show that the construction of extremal NSA-measures as established in [5] now follows as a special case. We assume that $g=0$.

A subfamily $\mathcal{S}$ of $\mathcal{C}_{\mathrm{s}}$ is called a co-basis if the following four conditions hold ([5], Definition 3.6):

(S1) $C_{1} \subseteq C_{2} \in \mathcal{S} \Rightarrow C_{1} \in \mathcal{S}$ if $C_{1} \in \mathcal{C}_{\mathrm{s}}$.

(S2) $C_{1}, C_{2} \in \mathcal{C}_{\mathrm{s}} \backslash \mathcal{S} \Rightarrow C_{1} \cap C_{2} \neq \emptyset$.

(S3) $C_{1}, C_{2} \in \mathcal{S} \Rightarrow C_{1} \cup C_{2} \neq X$.

(S4) For all $C_{1} \in \mathcal{S}$ there is a $C_{2} \in \mathcal{S}$ such that $C_{1} \subseteq C_{2}^{0}$.

Suppose $\mathcal{S}$ is a co-basis and define, for $C \in \mathcal{C}_{\mathrm{s}}$,

$$
\mu(C)= \begin{cases}0 & \text { if } C \in \mathcal{S}, \\ 1 & \text { if } C \notin \mathcal{S} .\end{cases}
$$

Let us verify that $\mu$ is a solid set-function. By (S1) it is clear that $\emptyset \in \mathcal{S}$, and by (S3) it follows that $X \notin \mathcal{S}$. Hence $\mu(X)=1$. Now suppose $C_{1}, C_{2} \in$ $\mathcal{C}_{\mathrm{s}}, C_{1} \cap C_{2}=\emptyset$. By (S2) at most one of these sets is not in $\mathcal{S}$. Combining this fact with (S1) we get (A). To get (B) it suffices to show that if $C_{1} \in \mathcal{S}$ then there is a set $C_{1}^{\prime} \in \mathcal{C}_{\mathrm{s}} \backslash \mathcal{S}$ such that $C_{1} \cap C_{1}^{\prime}=\emptyset$. By (S4) we get $C_{2} \in \mathcal{S}$ such that $C_{1} \subseteq C_{2}^{0}$. By Lemma 3.3 there is a set $W \in \mathcal{O}_{\mathrm{s}}$ such that $C_{1} \subseteq W \subseteq C_{2}^{0}$. Then $C_{1}^{\prime}=X \backslash W \in \mathcal{C}_{\mathrm{s}}$ and $C_{1}^{\prime} \cup C_{2}=X$ so by (S3) we must have $C_{1}^{\prime} \notin \mathcal{S}$. So $\mu$ is a solid set-function, and we get the following (Corollary 3.7 of [5]):

Proposition 5.2. $\mu$ as defined by (5.6) has a unique extension to an extremal NSA-measure in $X$.

A more general example may be given as follows: Let $P=\left\{p_{1}, \ldots, p_{q}\right\}$ be a set of $q$ distinct points in $X$, where

$$
q=2^{n+1}+1, \quad n \in \mathbb{Z}^{+} .
$$

For any set $C \in \mathcal{C}_{\mathrm{s}}$ let $\sharp C$ denote the number of elements in $P \cap C$. Define, for $k=0,1, \ldots, 2^{n}$,

$$
\mu_{n}(C)=k / 2^{n} \quad \text { if } \sharp C=2 k \vee 2 k+1 .
$$

We leave it to the reader to verify that $\mu_{n}$ is a solid set-function. It is easily seen that $\mu_{n}$ is not subadditive, so that the resulting NSA-measure is not (the restriction of) a regular Borel measure. However, one may show (using the same technique as in Example 2.1 of [2]) that each $\mu_{n}$ is an extreme point in the convex set $Q$ of all normalized NSA-measures in $X$.

We close this section with a "continuous example", i.e. a NSA-measure $\mu$ such that $\operatorname{Sp} \mu$ is all of $[0,1]$. Let $X=S^{2}=$ the unit sphere in $\mathbb{R}^{3}$, and let $\lambda$ denote normalized Lebesgue measure in $X$, i.e. $\lambda(X)=1$. Let $E=$ $\left\{(x, y, z) \in S^{2}: z=0\right.$, i.e. $\left.x^{2}+y^{2}=1\right\}=$ the equator on $S^{2}$. 
Define $\mu$ on $\mathcal{C}_{\mathrm{s}}$ as follows:

(i) $\mu(C)=1$ if $E \subseteq C$.

(ii) $\mu(C)=\lambda(C)$ if $E \cap C \neq \emptyset$ and $E \cap C^{\mathrm{c}} \neq \emptyset$.

(iii) $\mu(C)=0$ if $E \subseteq C^{\mathrm{c}}$.

Let us verify $(\mathrm{A})$ and $(\mathrm{B})$.

(A) Let $C_{1}, \ldots, C_{n}$ be a disjoint family in $\mathcal{C}_{\mathrm{s}}$, all contained in $C \in \mathcal{C}_{\mathrm{s}}$. We may assume that all the $C_{i}$ fall in the category (ii), otherwise there is nothing to prove. Then $\sum_{i=1}^{n} \mu\left(C_{i}\right)=\sum_{i=1}^{n} \lambda\left(C_{i}\right) \leq \lambda(C)$. Since $C \cap E \neq \emptyset$ the assertion follows.

(B) Let $C \in \mathcal{C}_{\mathrm{s}}$ and $\varepsilon>0$ be given. If $E \subseteq C$ there is nothing to prove, and if $E \subseteq C^{\mathrm{c}}$ we just take $C^{\prime}=E$. So assume that $E \cap C \neq \emptyset$ and $E \cap C^{\mathrm{c}} \neq \emptyset$. Since $C^{\mathrm{c}} \in \mathcal{O}_{\mathrm{s}}$, by Lemma 3.3 it follows that there is a set $C^{\prime} \in \mathcal{C}_{\mathrm{s}}$ such that $\lambda\left(C^{\prime}\right)+\lambda(C)>1-\varepsilon$, and $C^{\prime} \subseteq C^{\mathrm{c}}, C^{\prime} \cap E \neq \emptyset$. This establishes (B).

The NSA-measure constructed in this way is not a measure since it lacks subadditivity.

6. Discretization of measures. In this section we provide an affirmative solution to the following problem: Given a regular Borel measure $\lambda$ with $\operatorname{Sp} \lambda=[0,1]$, is it possible to find NSA-measures $\mu$ with finite spectra which approximate $\lambda$ arbitrarily well?

We shall assume that $X$ is a compact Hausdorff space which is connected, locally connected and have $g=0$. Since there is no need to assume that the initial measure $\lambda$ is really a measure, we just assume that $\lambda$ is a normalized NSA-measure.

Definition. Two disjoint sets $C_{1}, C_{2} \in \mathcal{C}_{\mathrm{s}}$ with non-zero $\lambda$-measure are called a splitting pair for $\lambda$ if $\lambda\left(C_{1}\right)+\lambda\left(C_{2}\right)=1$. $\lambda$ is non-splitting if there is no splitting pair for $\lambda$.

Now suppose $\lambda$ is a non-splitting NSA-measure satisfying $\lambda(X)=1$. Let $n$ be an arbitrary non-negative integer and put $q=2^{n}+1$. We are going to construct a NSA-measure $\mu_{n}$ such that $\operatorname{Sp} \mu_{n}=\left\{k / 2^{n}: k=0,1, \ldots, 2^{n}\right\}$. Define intervals $I_{k}$ as follows:

$$
\begin{aligned}
I_{k} & =[k / q,(k+1) / q), \quad k=0,1, \ldots, 2^{n}-1, \\
I_{2^{n}} & =\left[2^{n} / q, 1\right]
\end{aligned}
$$

We define, for $C \in \mathcal{C}_{\mathrm{s}}$,

$$
\mu_{n}(C)=k / 2^{n} \quad \text { if } \lambda(C) \in I_{k}, \quad \mu_{n}(X \backslash C)=1-\mu_{n}(C),
$$


Note that $\mu_{n}(C) \in I_{k}$ for all $k$,

$$
\begin{array}{ll}
\mu_{n}(C)=0 & \text { if } \lambda(C)<1 / q \text { and } \\
\mu_{n}(C)=1 & \text { if } \lambda(C) \geq 2^{n} / q .
\end{array}
$$

Proposition 6.1. $\mu_{n}$ is a solid set-function in $X$.

Proof. Let $C_{1}, \ldots, C_{m} \subseteq C \in \mathcal{C}_{\mathrm{s}}, C_{i} \in \mathcal{C}_{\mathrm{s}}$ for $i=1, \ldots, m$ and $C_{i} \cap C_{j}=\emptyset$ if $i \neq j$.

Case 1: $\lambda(C) \in I_{k}$ for some $k<2^{n}$. Then $\sum_{i=1}^{m} \lambda\left(C_{i}\right) \leq \lambda(C)<$ $(k+1) / q$. For $j=0,1, \ldots, 2^{n}$ let

$$
J_{j}=\left\{i: 1 \leq i \leq m, \lambda\left(C_{i}\right) \in I_{j}\right\}
$$

and let $n_{j}$ denote the number of elements in $J_{j}$. Since $C_{i} \subseteq C$ for all $i=$ $1, \ldots, m$ we have $n_{j}=0$ if $j>k$. Clearly $\sum_{j=0}^{k} n_{j}=m$. Now, if $\lambda\left(C_{i}\right) \in I_{j}$ then $\lambda\left(C_{i}\right) \geq j / q$, so we must have

$$
\frac{k+1}{q}>\sum_{j=1}^{m} \lambda\left(C_{j}\right) \geq \sum_{j=0}^{k} n_{j} \frac{j}{q}
$$

and so

$$
\sum_{j=1}^{k} j n_{j} \leq k
$$

Now $\mu_{n}\left(C_{i}\right)=j / 2^{n}$ if $i \in J_{j}$ so we get

$$
\begin{aligned}
\sum_{i=1}^{m} \mu_{n}\left(C_{i}\right) & =\sum_{j=1}^{k} \sum_{i \in J_{j}} \mu_{n}\left(C_{i}\right)=\sum_{j=1}^{k} n_{j} \frac{j}{2^{n}} \\
& =\frac{1}{2^{n}} \sum_{j=1}^{n} n_{j} j \leq \frac{k}{2^{n}}=\mu(C) .
\end{aligned}
$$

Case 2: $\lambda(C) \in I_{2^{n}}$. With notation as above we now get

$$
\sum_{j=1}^{2^{n}} j n_{j} \leq 2^{n}+1
$$

Suppose equality holds in (6.2). Then

$$
1 \geq \sum_{i=1}^{m} \lambda\left(C_{i}\right)=\sum_{j=1}^{2^{n}} \sum_{i \in J_{j}} \lambda\left(C_{i}\right) \geq \sum_{j=1}^{2^{n}} \frac{j}{2^{n}+1} n_{j}=1 .
$$


Hence we must have $\lambda\left(C_{i}\right)=j / q$ if $i \in J_{j}$. This violates the non-splitting of $\lambda$. We must therefore have

$$
\sum_{j=1}^{2^{n}} j n_{j} \leq 2^{n} .
$$

and the assertion $\sum_{i=1}^{m} \mu_{n}\left(C_{i}\right) \leq \mu_{n}(C)$ now follows as in Case 1. We have established (A).

Now let $C \in \mathcal{C}_{\mathrm{s}}$ be arbitrary, and assume that $\lambda(C) \in I_{k}$ for some $k<2^{n}$. (If $k=2^{n}$ then $\mu_{n}(C)=1$ and there is nothing to prove). Let $U=X \backslash C$ so $\lambda(U) \in((q-(k+1)) / q,(q-k) / q]$. By the regularity of $\lambda$ and Lemma 3.1 there is a set $C^{\prime} \subseteq U$ such that $C^{\prime} \in \mathcal{C}_{\mathrm{s}}$ and $\lambda\left(C^{\prime}\right) \in$ same interval. Then $\mu_{n}\left(C^{\prime}\right)=(q-(k+1)) /(q-1)$, since we cannot have $\lambda\left(C^{\prime}\right)=(q-k) / q$ because of the non-splitting of $\lambda$. But then

$$
\mu_{n}(C)+\mu_{n}\left(C^{\prime}\right)=\frac{k}{q-1}+\frac{q-(k+1)}{q-1}=1,
$$

which shows that $(\mathrm{B})$ is true. The proof is complete.

Proposition 6.2. Let $\lambda$ be a normalized non-splitting NSA-measure (or regular Borel measure) in $X$. For $n=1,2, \ldots$ let $\mu_{n}$ be defined by $(\mathrm{D})$. Then the resulting sequence $\left\{\mu_{n}\right\}$ of NSA-measures converges to $\lambda$ in the topology of $Q$.

Pr o o f. By definition it follows that $\left|\lambda(C)-\mu_{n}(C)\right|<1 / 2^{n}$ for all $C \in \mathcal{C}_{\mathrm{s}}$. Let $\left\{\mu_{n_{i}}\right\}$ be an arbitrary subsequence of $\left\{\mu_{n}\right\}$. Since $Q$ is compact, $\left\{\mu_{n_{i}}\right\}$ must have a subnet $\left\{\mu_{n_{i_{j}}}\right\}$ which converges to some element $\lambda^{\prime} \in Q$ (we identify $Q$ with the set of non-linear states). For $C \in \mathcal{C}_{\mathrm{s}}$ we then have

$$
\lambda^{\prime}(C)=\lim _{j} \mu_{n_{i_{j}}}(C)=\lambda(C)
$$

so $\lambda$ and $\lambda^{\prime}$ agree on $\mathcal{C}_{\mathrm{s}}$. But then, by uniqueness, we must have $\lambda=\lambda^{\prime}$. We have therefore shown that any subsequence of $\left\{\mu_{n}\right\}$ has a subnet which converges to $\lambda$. But then $\mu_{n}$ converges to $\lambda$. The proof is complete.

We do not know whether $\mu_{n}(K) \rightarrow \lambda(K)$ for all closed sets $K$ in $X$. What follows is what we can show, and what will suffice in most cases.

Proposition 6.3. If $C \in \mathcal{C}_{0}$ then $\mu_{n}(C) \rightarrow \lambda(C)$.

Proof. By definition we have $\mu_{n}(C)=k / 2^{n}$ if $\lambda(C) \in I_{k}(k=0,1, \ldots$ $\left.\ldots, 2^{n}\right)$ if $C \in \mathcal{C}_{\mathrm{s}}$. Let $I_{k}^{\prime}=(k / q,(k+1) / q]$ for $k=1, \ldots, 2^{n}$ and put $I_{0}^{\prime}=[0,1 / q]$. It is then easily verified that $\mu_{n}(V)=k / 2^{n}$ if $\lambda(V) \in I_{k}^{\prime}$ $\left(k=0,1, \ldots, 2^{n}\right)$ and $V \in \mathcal{O}_{\mathrm{s}}$. To prove the assertion it suffices to consider $C \in \mathcal{C}_{\mathrm{c}}$. Let such a $C \in \mathcal{C}_{\mathrm{c}}$ be given. We have $X \backslash C=V=\bigcup_{i \in I} V_{i}$, where the $V_{i}$ belong to $\mathcal{O}_{\mathrm{s}}$ and are disjoint. 
We now claim that for arbitrary $n \in \mathbb{Z}^{+}$we have (with $q_{n}=2^{n}+1$ )

$$
\sum_{i \in I} \mu_{n}\left(V_{i}\right)<\sum_{i \in I} \lambda\left(V_{i}\right)+\frac{1}{q_{n}}
$$

Indeed, let $J_{j}=\left\{i \in I: \lambda\left(V_{i}\right) \in I_{j}^{\prime}\right\}$ for $j=1, \ldots, 2^{n}$. For each $j \geq 1$ the number of elements in $J_{j}$ is finite and is denoted by $n_{j}$. If $\lambda(V) \in I_{k}^{\prime}$ we have $n_{j}=0$ for $j>k$. Since $\lambda\left(V_{i}\right)>j / q$ if $i \in J_{j}$ we now get

$$
\sum_{j=1}^{k} n_{j} \frac{j}{q}<\sum_{j=1}^{k} \sum_{i \in J_{j}} \lambda\left(V_{i}\right) \leq \sum_{i \in I} \lambda\left(V_{i}\right)=\lambda(V) \leq \frac{k+1}{q}
$$

so that $\sum n_{j} j \leq k$.

If $\lambda\left(V_{i}\right) \in I_{j}^{\prime}$ then $\mu_{n}\left(V_{i}\right)=j / 2^{n}$ so we get

$$
\sum_{i \in I} \mu_{n}\left(V_{i}\right)=\sum_{j=1}^{k} \sum_{i \in J_{j}} \mu_{n}\left(V_{i}\right)=\sum_{j=1}^{k} n_{j} \frac{j}{2^{n}} \leq \frac{k}{2^{n}}<\frac{k+1}{q} .
$$

Since $\sum_{i \in I} \lambda\left(V_{i}\right) \in I_{k}^{\prime}$ the claim (6.4) follows.

Now let $\varepsilon>0$ be arbitrary. Choose $N$ such that $\sum_{i>N} \lambda\left(V_{i}\right)<\varepsilon$. Then choose $N^{\prime}$ such that $1 /\left(2 N^{\prime}\right)<\varepsilon / N$. For $n \geq N^{\prime}$ we then obtain

$$
\sum_{i=1}^{N}\left|\lambda\left(V_{i}\right)-\mu_{n}\left(V_{i}\right)\right|<\frac{N}{2^{n}}<\varepsilon .
$$

Fix an arbitrary $n \geq N^{\prime}$. By (6.4) (applied to the family $\left\{V_{i}\right\}_{i>N}$ ) we get

$$
\sum_{i>N} \mu_{n}\left(V_{i}\right)<\sum_{i>N} \lambda\left(V_{i}\right)+\frac{1}{q_{n}}<\varepsilon+\frac{1}{q_{n}}<2 \varepsilon .
$$

Consequently, for any $n \geq N^{\prime}$,

$$
\begin{aligned}
\left|\lambda(C)-\mu_{n}(C)\right| & \leq \sum_{i \in I}\left|\lambda\left(V_{i}\right)-\mu_{n}\left(V_{i}\right)\right| \\
& \leq \sum_{i=1}^{N}\left|\lambda\left(V_{i}\right)-\mu_{n}\left(V_{i}\right)\right|+\sum_{i>N} \lambda\left(V_{i}\right)+\sum_{i>N} \mu_{n}\left(V_{i}\right) \\
& <\varepsilon+\varepsilon+2 \varepsilon=4 \varepsilon .
\end{aligned}
$$

Hence $\mu_{n}(C) \rightarrow \lambda(C)$ if $C \in \mathcal{C}_{\mathrm{c}}$. The proof is complete.

\section{References}

[1] J. F. Aarnes, Quasi-states and quasi-measures, Adv. in Math. 86 (1991), 41-67.

[2] - Pure quasi-states and extremal quasi-measures, Math. Ann. 295 (1993), 575-588.

[3] C. D. Christenson and W. L. Voxman, Aspects of Topology, Dekker, New York, 1977. 
[4] P. Halmos, Measure Theory, Van Nostrand, New York, 1950.

[5] F. F. Knudsen, Topology and the construction of extreme quasi-measures, Adv. in Math., to appear.

[6] H. L. Royden, Real Analysis, 2nd ed., Macmillan, New York, 1986.

UNIVERSITY OF TRONDHEIM, AVH

DEPARTMENT OF MATHEMATICS AND STATISTICS

7055 DRAGVOLL, NORWAY

Received 28 January 1994;

in revised form 27 January 1995 